\title{
IL-6 Inhibition Reduces STAT3 Activation and Enhances the Antitumor Effect of Carboplatin
}

\author{
Zhi-Yong Wang, 1,2 Jun-Ai Zhang, 1,2 Xian-Jin Wu,,3 Yan-Fang Liang, ${ }^{4}$ \\ Yuan-Bin Lu, ${ }^{1,2}$ Yu-Chi Gao, ${ }^{1,2}$ You-Chao Dai, ${ }^{1,2}$ Shi-Yan Yu, ${ }^{1,2}$ Yan Jia, ${ }^{1,2}$ \\ Xiao-Xia Fu, ${ }^{1}$ Xiaoquan Rao, ${ }^{5}$ Jun-Fa Xu, ${ }^{1,2}$ and Jixin Zhong ${ }^{5}$ \\ ${ }^{1}$ Guangdong Provincial Key Laboratory of Medical Molecular Diagnostics, No. 1 Xincheng Road, Dongguan 523808, China \\ ${ }^{2}$ Department of Clinical Immunology, Institute of Laboratory Medicine, Guangdong Medical University, No. 1 Xincheng Road, \\ Dongguan 523808, China \\ ${ }^{3}$ Department of Laboratory Medicine, Affiliated Hospital of Guangdong Medical University, Zhanjiang 524001, China \\ ${ }^{4}$ Department of Pathology, Dongguan 5th Hospital, Dongguan 523905, China \\ ${ }^{5}$ Department of Medicine, University of Maryland School of Medicine, Baltimore, MD 21201, USA
}

Correspondence should be addressed to Jun-Fa Xu; imxujunfa@163.com and Jixin Zhong; jzhong@medicine.umaryland.edu

Received 27 November 2015; Revised 27 January 2016; Accepted 1 February 2016

Academic Editor: Amedeo Amedei

Copyright (C) 2016 Zhi-Yong Wang et al. This is an open access article distributed under the Creative Commons Attribution License, which permits unrestricted use, distribution, and reproduction in any medium, provided the original work is properly cited.

\begin{abstract}
Recent studies suggest that tumor-associated macrophage-produced IL-6 is an important mediator within the tumor microenvironment that promotes tumor growth. The activation of IL-6/STAT3 axis has been associated with chemoresistance and poor prognosis of a variety of cancers including colorectal carcinoma and thus serves as a potential immunotherapeutic target for cancer treatment. However, it is not fully understood whether anticytokine therapy could reverse chemosensitivity and enhance the suppressive effect of chemotherapy on tumor growth. In this study, we aimed to investigate the effect of IL-6 inhibition therapy on the antitumor effect of carboplatin. Enhanced expression of IL- 6 and activation of STAT3 were observed in human colorectal carcinoma samples compared to normal colorectal tissue, with higher levels of IL-6/STAT3 in low grade carcinomas. Treatment of carboplatin (CBP) dose-dependently increased IL-6 production and STAT3 activation in human colorectal LoVo cells. Blockade of IL-6 with neutralizing antibody enhanced chemosensitivity of LoVo cells to carboplatin as evidenced by increased cell apoptosis. IL-6 blockade abolished carboplatin-induced STAT3 activation. IL-6 blockade and carboplatin synergistically reduced cyclin D1 expression and enhanced caspase-3 activity in LoVo cells. Our results suggest that inhibition of IL-6 may enhance chemosensitivity of colon cancers with overactive STAT3 to platinum agents.
\end{abstract}

\section{Introduction}

IL-6, produced by tumor-associated macrophage, is an important mediator that promotes tumor growth $[1,2]$. Although there was evidence supporting a role in T-cell activation and trafficking [3], IL-6 within the tumor microenvironment is generally considered as a malevolent player that promotes tumor progression. By activating downstream Janus kinase (JAK) signal transducer and activator of transcription-3 (STAT3) signaling, IL-6 promotes cancer cell proliferation, survival, and metastatic dissemination. In addition, IL-6 may also act on other cell types within the tumor microenvironment to enhance tumor growth by supporting angiogenesis [4] and immune escape [5, 6].

Platinum drugs such as cisplatin, carboplatin, and oxaliplatin are a class of chemotherapy agents that trigger apoptosis of tumor cells by binding to and causing DNA crosslinking. They are widely used in cancer chemotherapy due to their broad spectrum of activities against several solid tumors [7]. However, the drug resistance is a major problem in platinum-based therapy, with $75 \%$ relapse for cisplatin [8]. Enhanced activation of STAT3 has been suggested as a major contributor to platinum resistance $[9,10]$. In this investigation, we examine the effect of carboplatin (CBP) and 
IL-6 blockade combination therapy on the growth of LoVo, a human colon carcinoma cell line.

\section{Materials and Methods}

2.1. Human Colorectal Carcinoma Tissue Collection. Colorectal tumor and nontumor colon tissue samples were collected at the time of surgical resection at Dongguan 6th Hospital. All procedures involving human participants were approved by the Research Ethics Board and the Institutional Review Board (IRB) at the Guangdong Medical College and Dongguan 6th Hospital. Written informed consent was obtained before tissue collection.

2.2. Cell Culture and Reagents. The human colorectal cancer LoVo cells were purchased from ATCC (Manassas, VA, USA). LoVo cells were cultured in F12K medium supplemented with $10 \%$ fetal bovine serum, $100 \mathrm{~g} / \mathrm{mL}$ streptomycin, and $100 \mathrm{U} / \mathrm{mL}$ penicillin, at $37^{\circ} \mathrm{C}, 5 \% \mathrm{CO}_{2}$, and high humidity. The sources of antibodies (Abs) were as follows: IL-6 was purchased from R\&D (Minneapolis, MN, USA), p-STAT3 was purchased from Abcam (Cambridge, MA, USA), cleaved caspase- 3 was purchased from Cell Signaling (Beverly, MA, USA), and STAT3, cyclin D1, GAPDH, and the HRPlabeled secondary antibodies were purchased from EnoGene (Nanjing, China). Carboplatin was purchased from MelonePharma (Dalian, Liaoning, China). Annexin-V-FITC apoptosis detection kit, DAB Substrate Kit, and Cell Counting Kit-8 (CCK-8) were purchased from Beyotime (Beyotime, Shanghai, China). IL-6 ELISA kit was from NeoBioscience (Shenzhen, Guangdong, China).

2.3. Immunohistochemistry Detection. All human colorectal tumor and nontumor specimens were fixed in $10 \%$ neutralbuffered formalin, dehydrated in ascending series of ethanol, and routinely embedded in paraplast. Sections were cut at $10 \mu \mathrm{m}$ and overnight stained with indicated primary antibodies after deparaffinization, rehydration, antigen recovery, and blocking. After washing, sections were incubated with HRP-labeled corresponding secondary antibodies and the signal was developed with a DAB Substrate Kit (Beyotime, Shanghai, China).

2.4. ELISA Detection of IL-6. LoVo cells were treated with indicated concentration of CBP or vehicle for $48 \mathrm{~h}$. Culture supernatant was collected for the detection of IL- 6 using an ELISA kit (NeoBioscience) according to the manufacturer's instruction.

2.5. Apoptosis Detection. Cell apoptosis was measured by an Annexin-V-FITC apoptosis detection kit (Beyotime, Shanghai, China) following the manufacturer's instruction. Briefly, cells were incubated with $5 \mu \mathrm{Mol} / \mathrm{L}$ Annexin-V and $1 \mu \mathrm{g} / \mathrm{mL}$ propidium iodide (PI) at room temperature for $15 \mathrm{~min}$. Cells were then analyzed on a BD FACSCalibur cytometer within $1 \mathrm{~h}$.

2.6. Western Blot. Cells were lyzed after 24 hours of indicated treatment and subjected to western blot detection of
p-STAT3, STAT3, cyclin D1, cleaved caspase-3, and GAPDH as described [11]. Briefly, the blots were probed with an indicated primary Ab followed by an HRP-conjugated secondary antibody. The reactive bands were visualized using an ECL western blot kit.

\section{Results}

3.1. IL-6 Expression and STAT3 Activation in Human Colorectal Carcinoma. Increased expression of IL- 6 has been detected and associated with an unfavorable prognosis in patients with various types of cancers including breast cancer, colorectal carcinoma, and ovarian cancer [12-17]. To confirm whether IL-6-STAT3 axis is activated in colon cancer, human colorectal carcinoma and matched nontumor colon tissue samples were used for the immunohistochemistry detection of IL-6, phosphor-STAT3 (p-STAT3), and STAT3. As shown in Figure 1, IL-6 expression increased in colorectal carcinoma especially in low grade carcinoma. Consistent with this, both the activation of STAT3 and expression of total STAT3 were upregulated in colorectal carcinoma and higher levels were seen in low grade carcinoma (Figure 1(a)). These results were further confirmed by western blot detection. Levels of IL6 , STAT3, and p-STAT3 were all increased in the human colorectal carcinoma samples, with a higher increase in low grade carcinoma (Figure 1(b)).

\subsection{Effect of Carboplatin (CBP) Treatment on IL-6 Production} by LoVo Cells. Enhanced activation of STAT3 has been suggested to associate with the chemoresistance of platinum agents $[9,10]$. To examine whether CBP treatment could affect IL-6 production in tumor, LoVo cells were treated with different concentrations of CBP for $48 \mathrm{~h}$. Treatment with $1 \mu \mathrm{g} / \mathrm{mL}$ CBP did not affect IL-6 level in the culture supernatant, while both 5 and $20 \mu \mathrm{g} / \mathrm{mL}$ CBP treatments increased IL-6 production with a dose-dependent effect (Figure 2). Treatment of $20 \mu \mathrm{g} / \mathrm{mL}$ CBP resulted in an over 2 fold increase in IL- 6 secretion. Since IL- 6 has been suggested to promote tumor survival $[1,2]$, CBP-induced IL- 6 production might contribute to drug resistance to platinum.

3.3. Synergistic Effect of CBP and IL-6 Blockade on Colorectal Cancer Cell Apoptosis. Increased production of IL-6 and enhanced activation of STAT3 have been suggested to associate with platinum resistance $[9,10,18]$. To test the effect of IL-6 blockade on CBP chemosensitivity, cell viability and apoptosis of LoVo cells were examined 72 hours after treatment of IL- 6 neutralizing antibody (Ab) and/or CBP. As shown in Figure 3(a), a large amount of CBP-treated or IL-6Ab-treated cells changed their shape from a flat adherent to a rounded morphology, indicating an early stage of apoptosis. Combined treatment of IL- 6 blockade and CBP significantly enhanced this change with most of the cells showing apoptotic morphological changes. Consistent with that, cell viability assay by CCK- 8 confirmed that $\mathrm{CBP}$ treatment induced cell death/CCK-8 release and IL- 6 blockade further promoted cell death in CBP-treated LoVo cells (Figure 3(b)).

To further confirm the apoptosis induced by CBP and IL-6 blockade, LoVo cells after indicated treatments were 


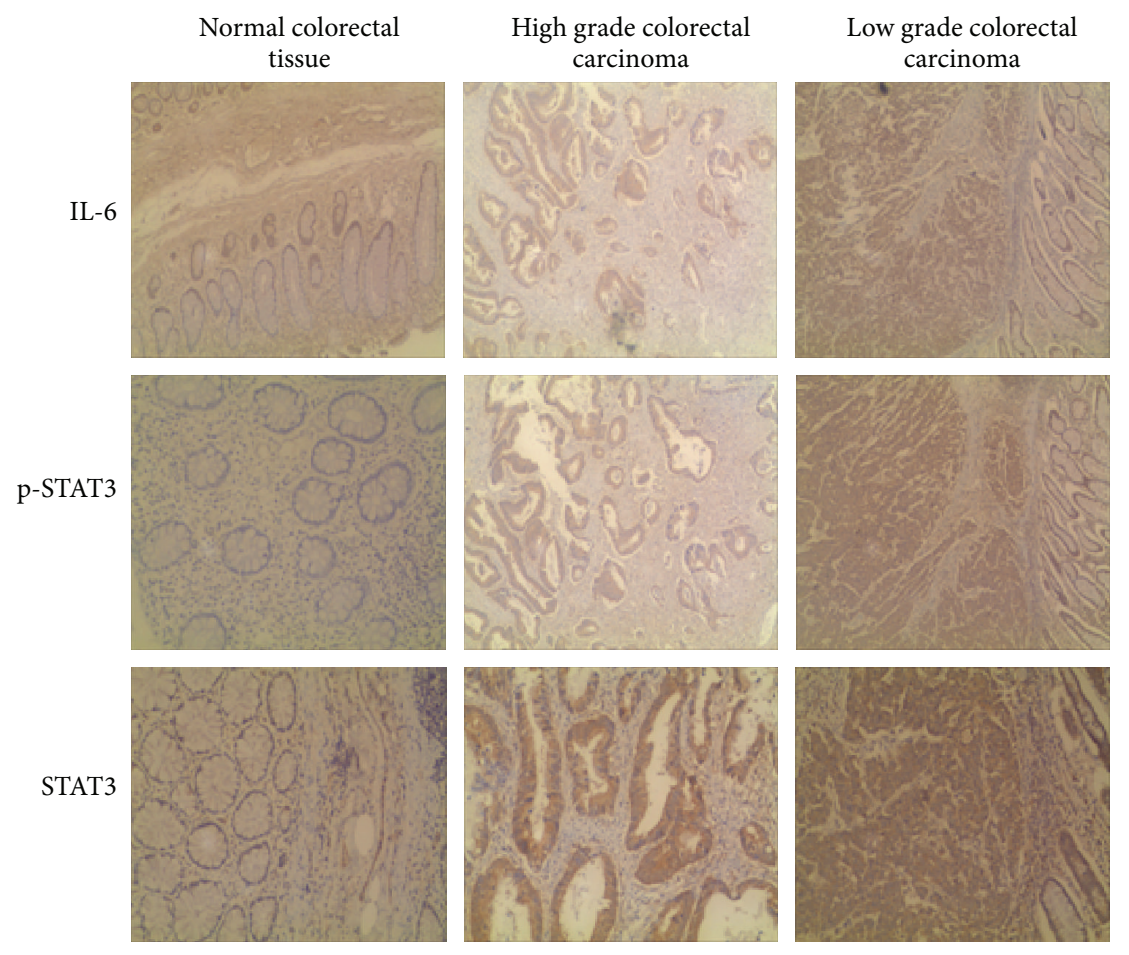

(a)

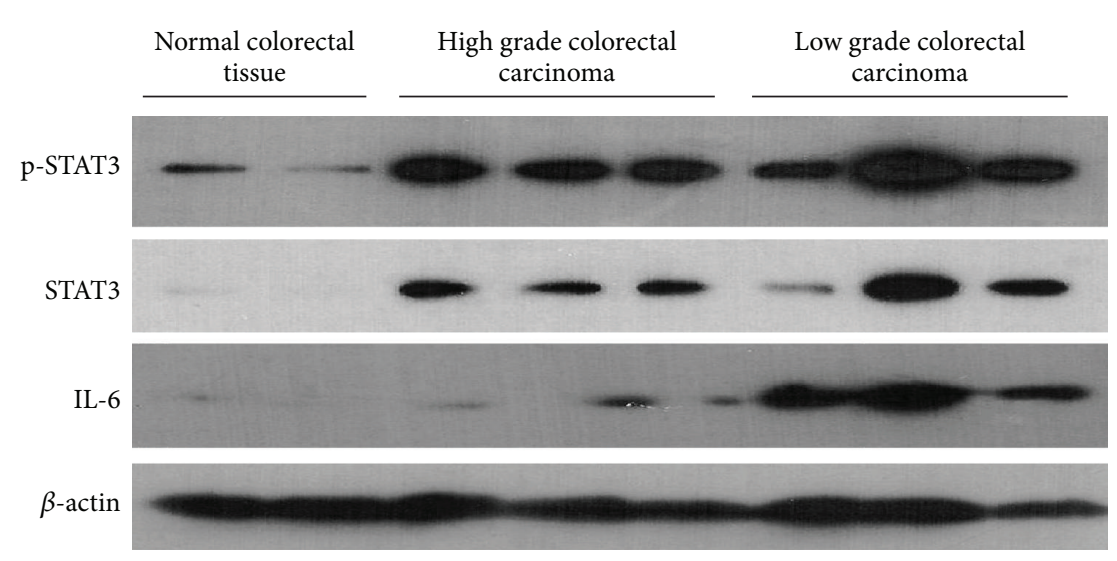

(b)

FIGURE 1: IL-6 expression and STAT3 activation in human colorectal carcinoma: (a) human colorectal carcinoma and nontumor samples were collected at surgery and paraffin-embedded sections were used for the immunohistochemistry detection of IL-6, p-STAT3, and STAT3. Representative images (200x magnification) were shown. (b) Western blot showing the expression of p-STAT3, STAT3, IL-6, and $\beta$-actin (internal control) in normal colorectal tissue, high grade colorectal carcinoma, and low grade colorectal carcinoma.

stained with Annexin-V and PI and analyzed on a flow cytometer. Results showed that both IL-6 Ab and CBP treatment increased apoptotic and necrotic cell number. Although frequencies of dead cells (cells in quadrants Q1, Q2, and Q4) were at a similar level in IL-6 Ab- and CBP-treated groups, the CBP-treated cells showed higher number of necrotic cells (quadrant Q1: 1.2\% versus 5.4\% versus $11.5 \%$ for cells treated with PBS versus IL-6 Ab versus CBP, resp.) while IL-6-treated cells displayed higher number of apoptotic cells (quadrant
Q4: 2.4\% versus 7.0\% versus 3.6\% for cells treated with PBS versus IL-6 Ab versus CBP, resp.). Combined treatment of IL-6 Ab + CBP dramatically enhanced both apoptosis $(1.2 \%$ versus $15.5 \%$ for PBS versus IL-6 Ab + CBP, Figure 4$)$ and necrosis $(2.4 \%$ versus $13.7 \%$ for PBS versus IL-6 Ab + CBP, Figure 4).

3.4. Effect of IL-6 Blockade and CBP on Pro- and Antisurvival Molecules. Western blot was then used to confirm 


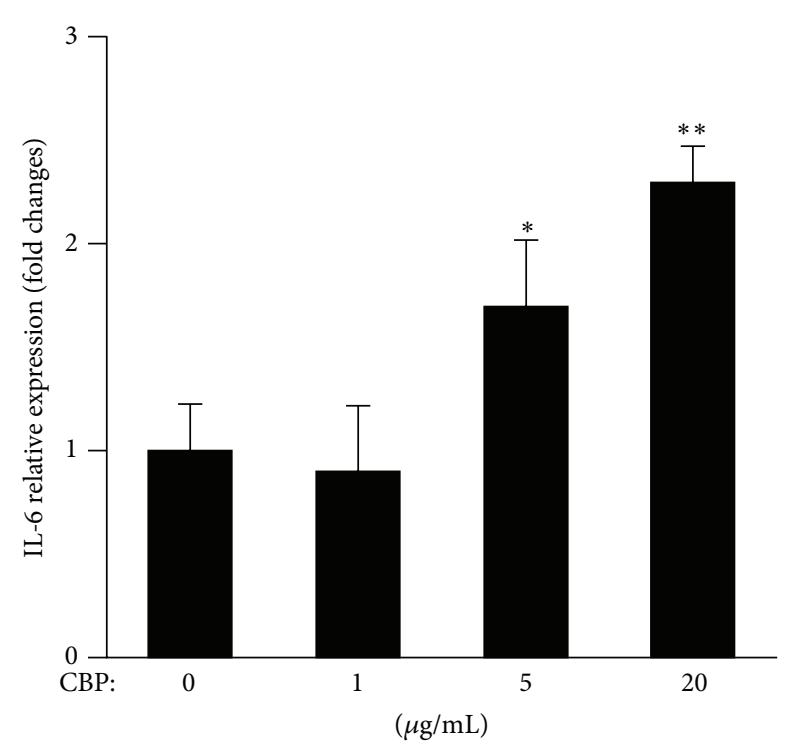

FIGURE 2: CBP treatment increased IL-6 production by LoVo cells: LoVo cells were treated with CBP at indicated doses for $48 \mathrm{~h}$. Culture supernatant was collected for ELISA detection of IL-6. ${ }^{*} P<0.05$; ${ }^{* *} P<0.01$.

the effect of CBP and IL- 6 blockade on STAT3 activation. Consistent with the increase of IL- 6 after CBP treatment, CBP enhanced the activation of STAT3 as evidenced by increased p-STAT3 level, while IL-6 blockade suppressed STAT3 activation (Figure 5). Cyclin D1, a well-described downstream target for STAT3 [19], was also increased by CBP and reduced by IL- 6 neutralization (Figure 5). Cleaved caspase- 3 is an apoptosis marker and is indispensable for cell apoptosis [20]. Both CBP and IL-6 blockade increased the activation of caspase-3, while combined treatment of IL-6 blockade and CBP further increased the activation of caspase3 (Figure 5).

\section{Discussion}

Combination therapy is the future direction for cancer treatment. More and more clinical evidence showed that monotherapy such as surgery, chemotherapy, or radiation therapy alone does not provide a satisfactory result for cancers. Chemotherapy is one of the most commonly used traditional therapies for cancer. However, studies indicate that most chemotherapy agents are detrimental to immunity. Therefore, immunotherapy or biologic therapy is increasingly used in combination with chemotherapy, a strategy referred to as "biochemotherapy" or "chemoimmunotherapy" [2125]. Of all the currently employed combination therapeutic strategies, platin-based regimen combined with biologic agents has attained the highest attention [26]. In the current study, we investigate the effect of anti-IL-6 therapy on CBP therapy.

IL-6 belongs to a cytokine family signaling through a common receptor gp130. The binding of IL- 6 to its receptor results in the activation of the associated Janus kinases
(JAKs), followed by the recruitment and activation of STAT3 and STAT1 [27]. Although an in vitro study suggests that IL6 suppresses in vitro growth of some cancer cells [28], IL6/STAT3 has been shown to promote tumor progression and immune escape in a variety of in vivo models [4-6]. Deficiency in STAT3 has been shown to protect against colitisassociated colorectal cancer in mice [29]. Therefore, IL6/STAT3 has been suggested as a potential immunotherapeutic target for malignant diseases $[6,30]$. By using immunohistochemistry, western blot, and ELISA, we found in this study that IL-6 is upregulated in colorectal carcinoma especially in low grade carcinoma, accompanied with enhanced STAT3 activation. In addition to active STAT3 (p-STAT3), total STAT3 protein level was also increased in both high grade and low grade colorectal carcinomas. This is probably because long term activation of STAT3 by IL-6 enhances the transcription and expression of STAT3. This result is consistent with the findings by other groups that IL- 6 increased during tumorigenesis [31, 32].

Carboplatin, also known as cis-diammine(1,1-cyclobutanedicarboxylato)platinum(II), is used as an anticancer chemotherapy drug for a variety of cancer types due to its broad spectrum of activities against several solid tumors [7]. It triggers tumor cell apoptosis by causing DNA crosslinking. However, its use in clinic is largely limited by the high incidence of drug resistance. Studies suggested that the activation of STAT3 is enhanced in platinum therapy and is responsible for platinum resistance $[9,10]$. We therefore hypothesize that IL-6 inhibition could improve platinum resistance. We first confirmed that CBP dose-dependently increased IL- 6 production by LoVo cells. IL- 6 blockade had a synergistic effect on promoting tumor cell apoptosis when combined with CBP.

CBP has been shown to induce cancer cell apoptosis via activation of caspases [33]. We verified this effect by detecting the viability and apoptosis rate. We also examined the effect of IL-6 blockade on tumor cell apoptosis with or without the presence of CBP. Our results suggest that IL- 6 blockade alone reduces tumor cell viability and promotes apoptosis. Furthermore, IL-6 blockade and CBP have synergistic effect on promoting apoptosis.

As a transcription factor, STAT3 mediates the expression of various genes including Bcl-XL, survivin, and cyclin D1 $[34,35]$. In our study, we confirmed that both IL-6 blockade and CBP activate caspase-3. Combined treatment of IL6 blockade and CBP had a synergistic effect on caspase-3 activation. CBP therapy enhanced STAT3 activation while IL6 blockade eliminated STAT3 activation in both CBP-treated LoVo cells and vehicle control. In consistency with that, cyclin D1 slightly increased in CBP-treated cells, while IL- 6 blockade dramatically diminished cyclin D1 expression in both CBPtreated LoVo cells and vehicle control. These suggest that IL6 blockade may block the adverse effect of CBP-induced IL6 upregulation and it has a synergistic effect on enhancing proapoptotic signaling.

In summary, our results indicate that IL- 6 blockade may enhance the antitumor effect of CBP and eliminate the adverse effects caused by CBP-induced IL- 6 upregulation. 

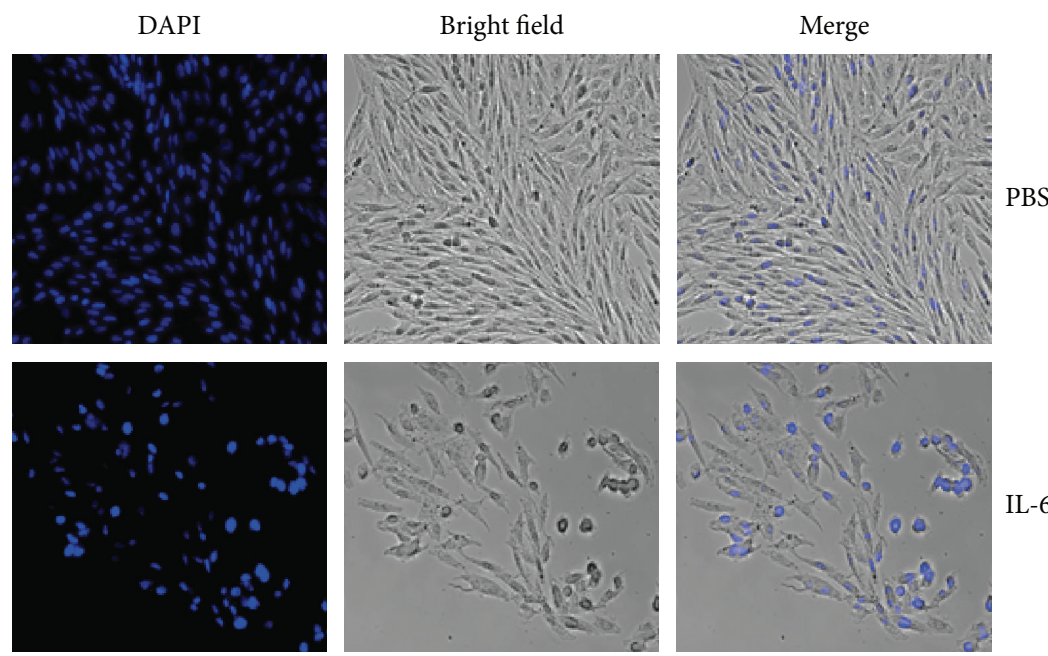

IL-6 Ab
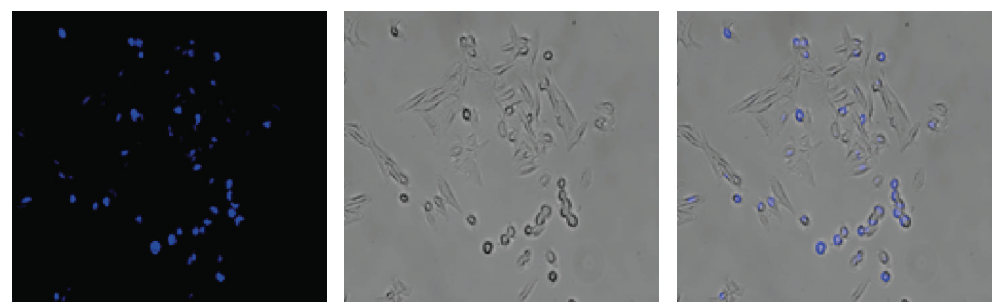

CBP
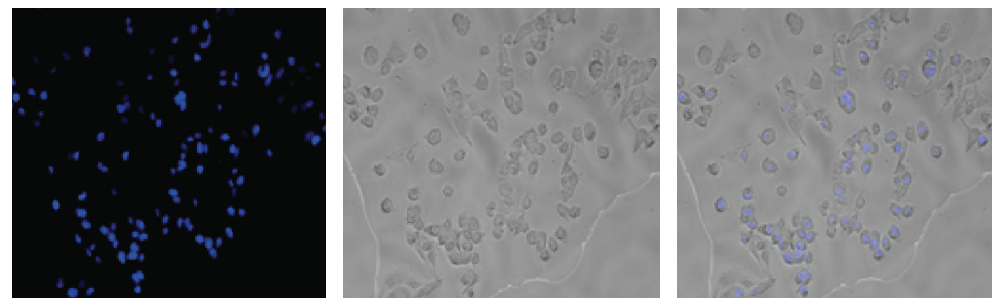

\section{BS}

(a)

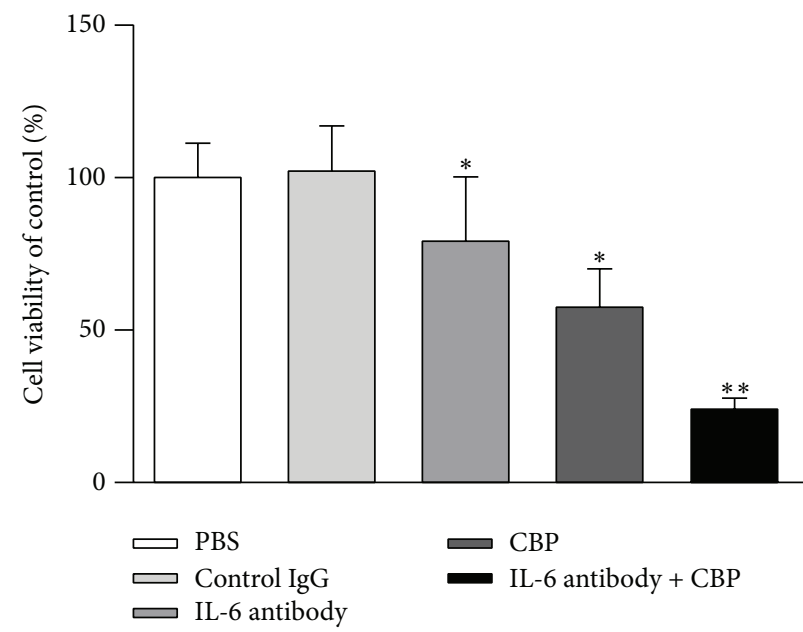

(b)

FIGURE 3: Synergistic effect of CBP and IL-6 blockade on LoVo cell survival: (a) LoVo cells were treated with $20 \mu \mathrm{g} / \mathrm{mL}$ CBP and/or $500 \mu \mathrm{g} / \mathrm{mL}$ IL-6 neutralizing antibody (Ab) or PBS. Cells were stained with DAPI $(0.2 \mu \mathrm{g} / \mathrm{mL})$ and morphological change was examined after $72 \mathrm{~h}$ under microscope (100x). (b) LoVo cells were treated with $20 \mu \mathrm{g} / \mathrm{mL}$ CBP and/or $500 \mu \mathrm{g} / \mathrm{mL}$ IL-6 neutralizing antibody (Ab) or PBS. Cells were collected for the detection of viability using CCK- 8 kit. ${ }^{*} P<0.05 ;{ }^{* *} P<0.01$. 

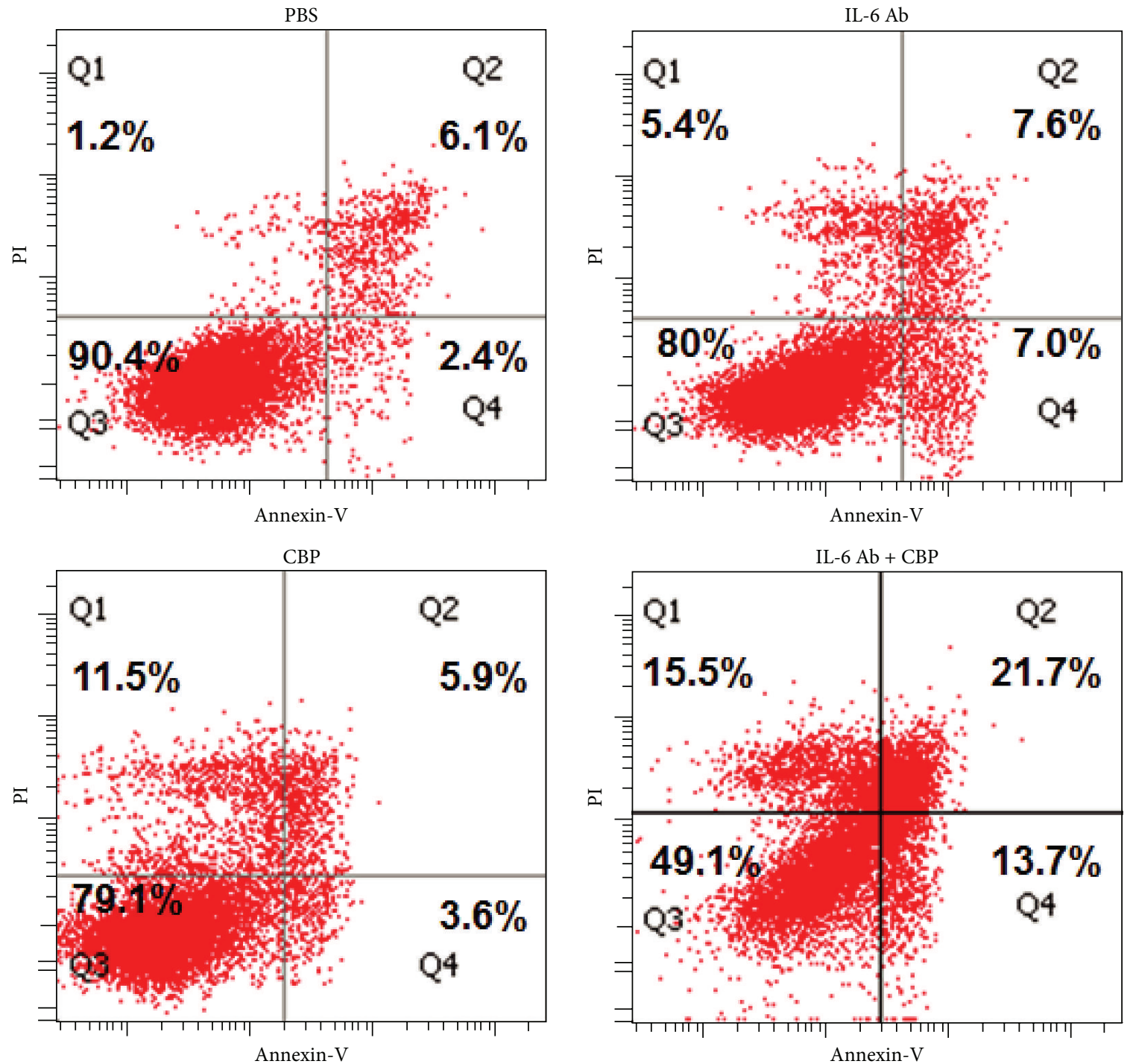

Figure 4: Effect of CBP and IL-6 blockade on LoVo cell apoptosis: LoVo cells were treated with $20 \mu \mathrm{g} / \mathrm{mL}$ CBP, $500 \mu \mathrm{g} / \mathrm{mL}$ IL-6 neutralizing antibody (Ab), or both combined or PBS for $24 \mathrm{~h}$. After staining with Annexin-V-FITC and PI, cells were analyzed on a flow cytometer.

However, further studies are required to confirm this effect in vivo.

\section{Conflict of Interests}

The authors report no conflict of interests in this work.

\section{Authors' Contribution}

Zhi-Yong Wang, Jun-Ai Zhang, and Yan-Fang Liang performed experiments and acquisition of data. Zhi-Yong Wang, Yuan-Bin Lu, Yu-Chi Gao, You-Chao Dai, Shi-Yan Yu, Yan Jia, and Xiao-Xia Fu interpreted the results and analyzed the data. Jun-Fa Xu and Jixin Zhong contributed to the study design. The paper was written by Xiaoquan $\mathrm{Rao}$, Jun-Fa $\mathrm{Xu}$, and
Jixin Zhong. All authors have read and approved the paper. Zhi-Yong Wang, Jun-Ai Zhang, and Xian-Jin Wu contributed equally to this work.

\section{Acknowledgments}

This work was supported by the National Natural Science Foundation of China (81101553, 30971779, and 81273237). Jixin Zhong was supported by grants from NIDDK/NIH (K01 DK105108) and AHA (15SDG25700381 and 13POST17210033). Jun-Fa Xu was supported by grants from the Key Project of Science and Technology Innovation of Education Department of Guangdong Province (2012KJCX0059), the Science and Technology Project of Dongguan (201450715200503), the Science and Technology Project of Zhanjiang 


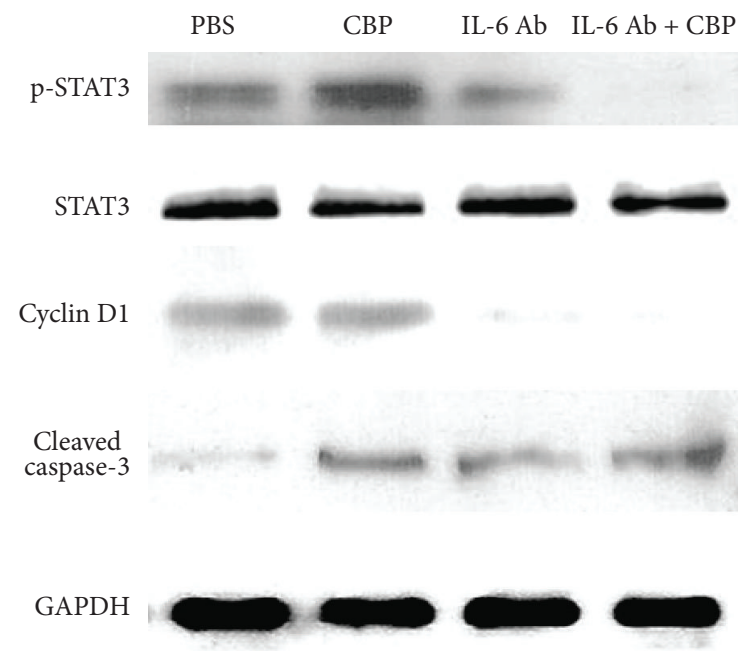

FIGURE 5: Western blot analysis of STAT3 and downstream molecules: LoVo cells were treated with $20 \mu \mathrm{g} / \mathrm{mL}$ CBP, $500 \mu \mathrm{g} / \mathrm{mL}$ IL-6 neutralizing antibody $(\mathrm{Ab})$, or both combined or PBS control for $24 \mathrm{~h}$. Total proteins were then isolated for the western blot detection of p-STAT3, total STAT3, cyclin D1, cleaved caspase-3, and GAPDH.

(2013C03012), and the Science and Technology Innovation Fund of GDMC (STIF201110, B2012078, and M2013046).

\section{References}

[1] A. Mantovani, P. Allavena, A. Sica, and F. Balkwill, "Cancerrelated inflammation," Nature, vol. 454, no. 7203, pp. 436-444, 2008.

[2] Q. Liao, Z. Zeng, X. Guo et al., "LPLUNC1 suppresses IL-6induced nasopharyngeal carcinoma cell proliferation via inhibiting the Stat 3 activation," Oncogene, vol. 33, no. 16, pp. 20982109, 2014.

[3] D. T. Fisher, Q. Chen, J. J. Skitzki et al., "IL-6 trans-signaling licenses mouse and human tumor microvascular gateways for trafficking of cytotoxic T cells," The Journal of Clinical Investigation, vol. 121, no. 10, pp. 3846-3859, 2011.

[4] T. Nagasaki, M. Hara, H. Nakanishi, H. Takahashi, M. Sato, and H. Takeyama, "Interleukin-6 released by colon cancer-associated fibroblasts is critical for tumour angiogenesis: anti-interleukin-6 receptor antibody suppressed angiogenesis and inhibited tumour-stroma interaction," British Journal of Cancer, vol. 110, no. 2, pp. 469-478, 2014.

[5] D. T. Fisher, M. M. Appenheimer, and S. S. Evans, "The two faces of IL-6 in the tumor microenvironment," Seminars in Immuno$\log y$, vol. 26, no. 1, pp. 38-47, 2014.

[6] H. Yu, H. Lee, A. Herrmann, R. Buettner, and R. Jove, "Revisiting STAT3 signalling in cancer: new and unexpected biological functions," Nature Reviews Cancer, vol. 14, no. 11, pp. 736-746, 2014.

[7] C. P. Belani, "Recent updates in the clinical use of platinum compounds for the treatment of lung, breast, and genitourinary tumors and myeloma," Seminars in Oncology, vol. 31, no. 14, pp. 25-33, 2004.
[8] R. E. Bristow and D. S. Chi, "Platinum-based neoadjuvant chemotherapy and interval surgical cytoreduction for advanced ovarian cancer: a meta-analysis," Gynecologic Oncology, vol. 103, no. 3, pp. 1070-1076, 2006.

[9] N. Borsellino, A. Belldegrun, and B. Bonavida, "Endogenous interleukin 6 is a resistance factor for cis-diamminedichloroplatinum and etoposide-mediated cytotoxicity of human prostate carcinoma cell lines," Cancer Research, vol. 55, no. 20, pp. 46334639, 1995.

[10] Z. Duan, R. Foster, D. A. Bell et al., "Signal transducers and activators of transcription 3 pathway activation in drug-resistant ovarian cancer," Clinical Cancer Research, vol. 12, no. 17, pp. 5055-5063, 2006.

[11] J. Zhong, P. Yang, K. Muta et al., "Loss of Jak2 selectively suppresses DC-mediated innate immune response and protects mice from lethal dose of LPS-induced septic shock," PLoS ONE, vol. 5, no. 3, Article ID e9593, 2010.

[12] G.-J. Zhang and I. Adachi, "Serum interleukin-6 levels correlate to tumor progression and prognosis in metastatic breast carcinoma," Anticancer Research, vol. 19, no. 2, pp. 1427-1432, 1999.

[13] M. Plante, S. C. Rubin, G. Y. Wong, M. G. Federici, C. L. Finstad, and G. A. Gastl, "Interleukin-6 level in serum and ascites as a prognostic factor in patients with epithelial ovarian cancer," Cancer, vol. 73, no. 7, pp. 1882-1888, 1994.

[14] M. B. Nilsson, R. R. Langley, and I. J. Fidler, "Interleukin-6, secreted by human ovarian carcinoma cells, is a potent proangiogenic cytokine," Cancer Research, vol. 65, no. 23, pp. 10794-10800, 2005.

[15] J. Kaminska, M. P. Nowacki, M. Kowalska et al., "Clinical significance of serum cytokine measurements in untreated colorectal cancer patients: soluble tumor necrosis factor receptor type Ian independent prognostic factor," Tumor Biology, vol. 26, no. 4, pp. 186-194, 2005.

[16] J. Shimazaki, Y. Goto, K. Nishida et al., "In patients with colorectal cancer, preoperative serum interleukin-6 level and granulocyte/lymphocyte ratio are clinically relevant biomarkers of long-term cancer progression," Oncology, vol. 84, no. 6, pp. 356-361, 2013.

[17] F. Esfandi, S. Mohammadzadeh Ghobadloo, and G. Basati, "Interleukin-6 level in patients with colorectal cancer," Cancer Letters, vol. 244, no. 1, pp. 76-78, 2006.

[18] S. Cohen, I. Bruchim, D. Graiver et al., "Platinum-resistance in ovarian cancer cells is mediated by IL- 6 secretion via the increased expression of its target cIAP-2," Journal of Molecular Medicine, vol. 91, no. 3, pp. 357-368, 2013.

[19] K. Leslie, C. Lang, G. Devgan et al., "Cyclin D1 is transcriptionally regulated by and required for transformation by activated signal transducer and activator of transcription 3," Cancer Research, vol. 66, no. 5, pp. 2544-2552, 2006.

[20] A. G. Porter and R. U. Jänicke, "Emerging roles of caspase-3 in apoptosis," Cell Death and Differentiation, vol. 6, no. 2, pp. 99104, 1999.

[21] D. T. Alexandrescu, J. P. Dutcher, and P. H. Wiernik, "Metastatic melanoma: is biochemotherapy the future?" Medical Oncology, vol. 22, no. 2, pp. 101-111, 2005.

[22] M. B. Atkins, "Cytokine-based therapy and biochemotherapy for advanced melanoma," Clinical Cancer Research, vol. 12, no. 7, pp. 2353s-2358s, 2006.

[23] L. A. Emens, "Chemoimmunotherapy," Cancer Journal, vol. 16, no. 4, pp. 295-303, 2010. 
[24] J. Delgado, T. Baumann, G. Ghita, and E. Montserrat, "Chronic lymphocytic leukemia therapy: beyond chemoimmunotherapy," Current Pharmaceutical Design, vol. 18, no. 23, pp. 33563362, 2012.

[25] C. S. Tam and M. J. Keating, "Chemoimmunotherapy of chronic lymphocytic leukemia," Nature Reviews Clinical Oncology, vol. 7, no. 9, pp. 521-532, 2010.

[26] S. Verma, T. Petrella, C. Hamm, K. Bak, and M. Charette, "Biochemotherapy for the treatment of metastatic malignant melanoma: a clinical practice guideline," Current Oncology, vol. 15, no. 2, pp. 85-89, 2008.

[27] P. C. Heinrich, I. Behrmann, S. Haan, H. M. Hermanns, G. Müller-Newen, and F. Schaper, "Principles of interleukin (IL)6-type cytokine signalling and its regulation," The Biochemical Journal, vol. 374, no. 1, pp. 1-20, 2003.

[28] H. Knüpfer and R. Preiß, "Significance of interleukin-6 (IL-6) in breast cancer (review)," Breast Cancer Research and Treatment, vol. 102, no. 2, pp. 129-135, 2007.

[29] S. Grivennikov, E. Karin, J. Terzic et al., "IL-6 and stat3 are required for survival of intestinal epithelial cells and development of colitis-associated cancer," Cancer Cell, vol. 15, no. 2, pp. 103-113, 2009.

[30] M. Lesina, S. M. Wörmann, P. Neuhöfer, L. Song, and H. Algül, "Interleukin-6 in inflammatory and malignant diseases of the pancreas," Seminars in Immunology, vol. 26, no. 1, pp. 80-87, 2014.

[31] F. R. Greten, L. Eckmann, T. F. Greten et al., "IKK $\beta$ links inflammation and tumorigenesis in a mouse model of colitis-associated cancer," Cell, vol. 118, no. 3, pp. 285-296, 2004.

[32] W. E. Naugler, T. Sakurai, S. Kim et al., "Gender disparity in liver cancer due to sex differences in MyD88-dependent IL-6 production," Science, vol. 317, no. 5834, pp. 121-124, 2007.

[33] M. Itoh, H. Chiba, T. Noutomi, E. Takada, and J. Mizuguchi, "Cleavage of Bax- $\alpha$ and Bcl-x(L) during carboplatin-mediated apoptosis in squamous cell carcinoma cell line," Oral Oncology, vol. 36, no. 3, pp. 277-285, 2000.

[34] A. Xiong, Z. Yang, Y. Shen, J. Zhou, and Q. Shen, “Transcription factor STAT3 as a novel molecular target for cancer prevention," Cancers, vol. 6, no. 2, pp. 926-957, 2014.

[35] R. L. Carpenter and H.-W. Lo, "STAT3 target genes relevant to human cancers,” Cancers, vol. 6, no. 2, pp. 897-925, 2014. 


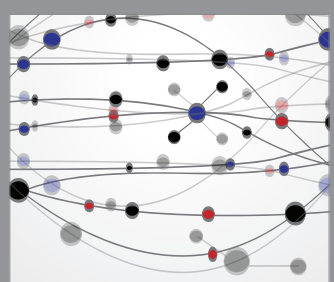

The Scientific World Journal
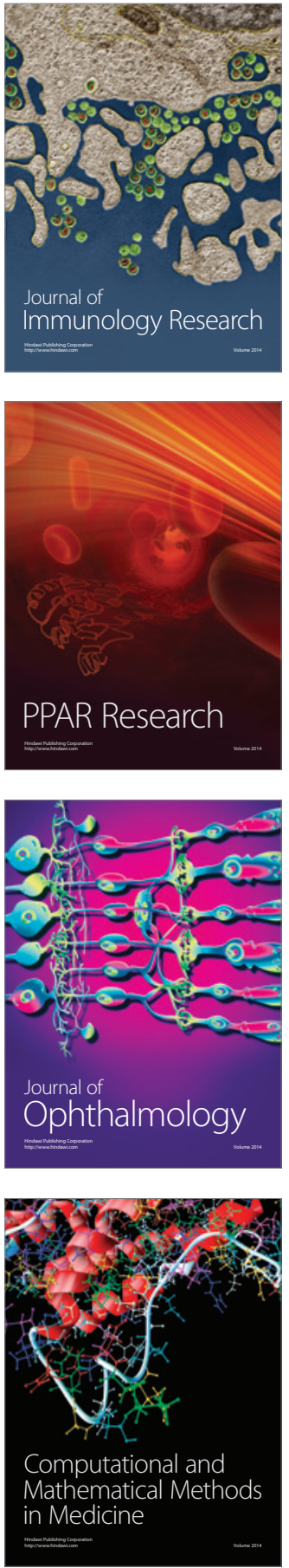

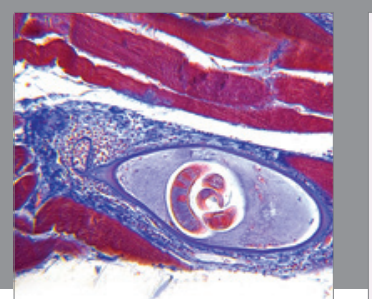

Gastroenterology Research and Practice

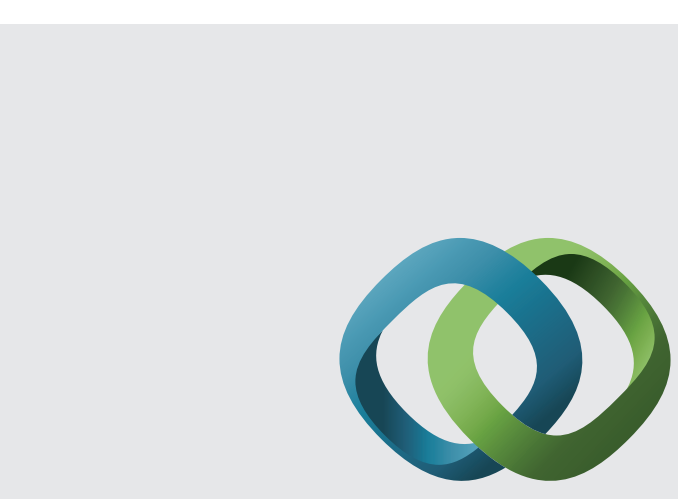

\section{Hindawi}

Submit your manuscripts at

http://www.hindawi.com
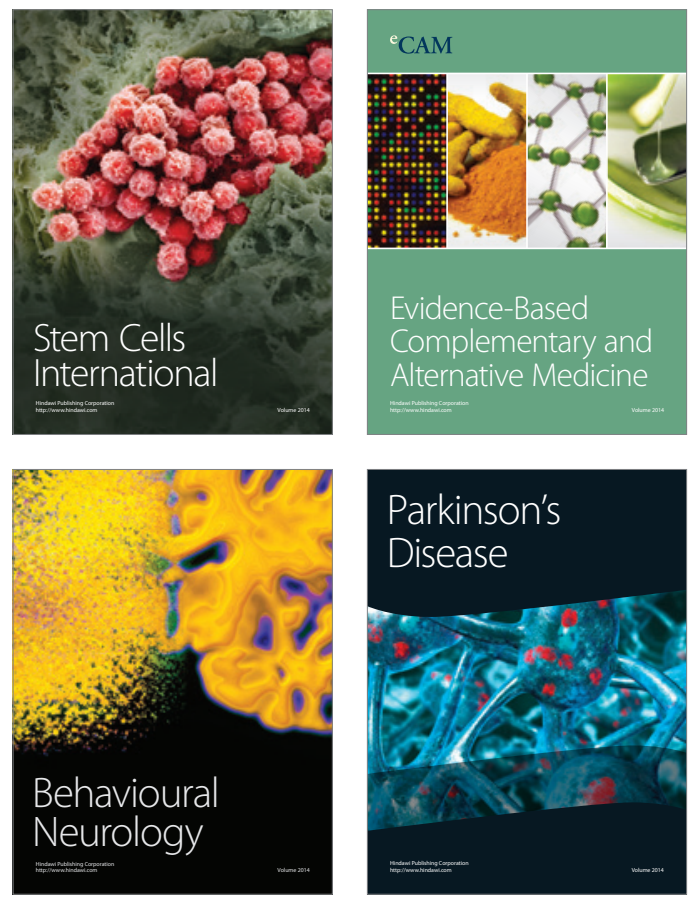
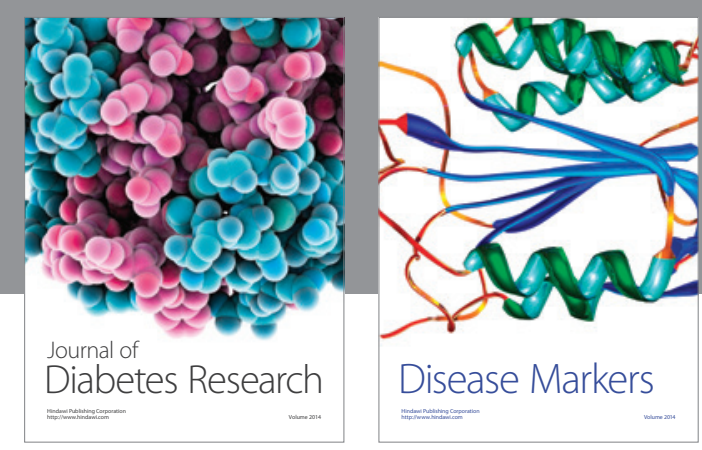

Disease Markers
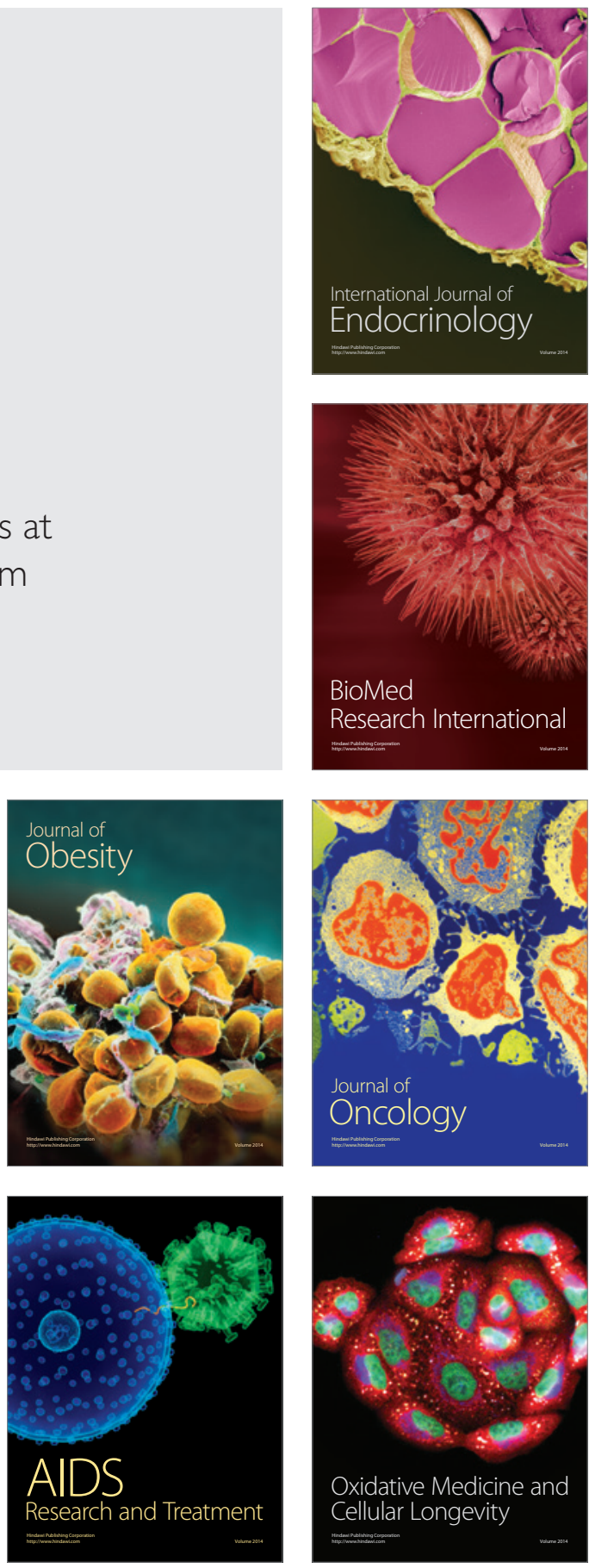